\title{
Early detection of excessive stress in people due to the ongoing COVID-19 pandemic: studies including those using biological markers
}

\author{
Yasuyuki Fujita ${ }^{1} \oplus$, Ken Inoue ${ }^{2} \oplus$, Nursultan Seksenbayev ${ }^{3}$, Nailya Chaizhunusova ${ }^{3}$, Masaharu Hoshi $^{4}{ }^{\oplus}$,

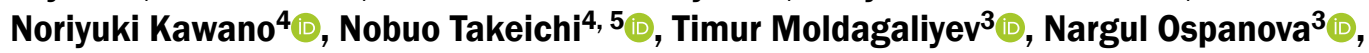

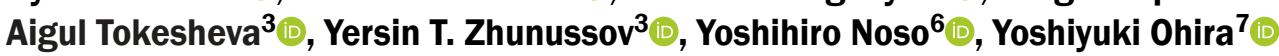 \\ ${ }^{1}$ Shimane University, Shimane, Japan \\ ${ }^{2}$ Kochi University, Kochi, Japan \\ ${ }^{3}$ Semey Medical University, Semey, Kazakhstan \\ ${ }^{4}$ Hiroshima University, Hiroshima, Japan \\ ${ }^{5}$ Takeichi Clinic, Hiroshima, Japan \\ ${ }^{6}$ Hiroshima International University, Hiroshima, Japan \\ ${ }^{7}$ International University of Health and Welfare, School of Medicine, Chiba, Japan
}

The coronavirus disease 2019 (COVID-19) epidemic continues to affect countries around the world. As of May 22, 2021, there were about 33 million people in the United States infected with severe acute respiratory syndrome coronavirus 2 (SARS-CoV-2) (the virus that causes COVID-19), about 26 million in India, about 16 million in Brazil, about 5.9 million in France, about 5.2 million in Turkey, about 4.9 million in Russia, and about 720,000 in Japan [1, 2]. SARS-CoV-2 variants are also prevalent now, and their spread will presumably lead to a great deal of stress. The ongoing effects of the COVID-19 pandemic on various aspects of people's lives will also presumably intensify stress. Excessive stress needs to be quickly detected and dealt with. Detection of stress is often difficult, so this report has been conducted from the perspective of ascertaining and dealing with stress.

Discussion of stress involves use of 1) a questionnaire like that used in a "stress check" system [3], 2) a questionnaire about everyday life, and 3) biological markers. Discussion using the approach in 1): examine stress factors at work, the mental and physical stress response, and support from others. Discussion using the approach in 2): look at sleep, eating a regular diet, addiction to smartphones and video games, an individual's physical and mental state, ties to friends and family (is the individual isolated?), school attendance, and whether the individual is receiving needed outpatient care and taking his/her medication. Discussion looking at 3): typically use cortisol and amylase [4, 5] as stress markers and chromogranin $A$ as a mental stress marker.

This report has detected excessive stress early on using these three approaches. However, personnel in various areas such as members of the public, family members, members of the community, schools, the workplace, and medical personnel need to work together to deal with stress caused by the ongoing COVID-19 pandemic so that it does not result in mental or physical illness or lead to suicidal behaviour.

\section{FUNDING AND ACKNOWLEDGMENTS}

This work was supported by JSPS KAKENHI Grant-in-Aid for Scientific Research (C) Number 17K09194 awarded to K.I., JSPS KAKENHI Grant-in-Aid for Scientific Research (A) Number $19 H 01149$ awarded to M.H., JSPS KAKENHI Grantin-Aid for Scientific Research (B) Number 19H04355 awarded to N.K., and JSPS KAKENHI Grant-in-Aid for Scientific Research (C) Number $21 \mathrm{~K} 02383$ awarded to K.I. 


\section{REFERENCES}

1. NHK. https://www3.nhk.or.jp/news/special/coronavirus/world-data/ (cited 2021 May 22).

2. NHK. https://www3.nhk.or.jp/news/special/coronavirus/data-all/ (cited 2021 May 22).

3. Ministry of Health, Labour and Welfare. https://www.mhlw.go.jp/ bunya/roudoukijun/anzeneisei12/pdf/150709-1.pdf (cited 2021 May 16).
4. Inoue K, Hashioka S, Takeshita $\mathrm{H}$, et al. High serum cortisol levels as a potential indicator for changes in well-regulated daily life among junior high school students. Tohoku J Exp Med. 2019; 249(3): 143-146, doi: 10.1620/tjem.249.143, indexed in Pubmed: 31685782.

5. leda M, Miyaoka T, Wake R, etal. Evaluation of autonomic nervous system by salivary alpha-amylase level and heart rate variability in patients with schizophrenia. Eur Arch Psychiatry Clin Neurosci. 2014; 264(1): 83-87, doi: 10.1007/s00406-013-0411-6, indexed in Pubmed: 23645102. 